\title{
The Value of Intraoperative Sonographic Evaluation of Neck in Primary Hyperparathyroidsm: Case Report of Preoperative Double Adenoma Upgraded to Triple Intraoperatively
}

Fatih Kuzul, Dilek Arpaci, Guldeniz Karadeniz Cakmak², Rabia Uslü, Ali Ugur Emre ${ }^{2}$ Sevil Ilikhan Uygun 4 , Mehmet Cabuk ${ }^{3}$, Taner Bayraktaroglu ${ }^{1}$

${ }^{1}$ Division of Endocrinology and Metabolism, Department of Internal Medicine, Faculty of Medicine, Bulent Ecevit University, Zonguldak, Turkey ${ }^{2}$ Department of General Surgery, Faculty of Medicine, Bulent Ecevit University, Zonguldak, Turkey;

${ }^{3}$ Department of Nuclear Medicine, Faculty of Medicine, Bulent Ecevit University, Zonguldak, Turkey

${ }^{4}$ Department of Internal Medicine, Faculty of Medicine, Bulent Ecevit University, Zonguldak, Turkey;

\section{Background}

Primary hyperparathyroidism (PHPT), caused by increased parathyroid hormone (PTH) secretion, leads to generalized disorder of bone metabolism characterized with hypercalcemia and hypophosphatemia. Causes of PHPT include solitary parathyroid adenoma (80\%), primary parathyroid hyperplasia (10-15\%), parathyroid carcinoma (1-2\%). Other rare cause is double parathyroid adenomas (DPA) with frequency less than $1-2 \%$, which can be sporadic or familial. DPA should be considered for persistent or recurrent PHTP cases.

\section{Case Report}

A forty-five years old female patient with Type 2 diabetes was admitted with weakness, fatigue and generalized bone pain. She had a history nephrolithiasis five times in the last two years. In laboratory examination, serum calcium level was $12.3 \mathrm{mg} / \mathrm{dL}$ (8.8-10.6), phosphorus level was 2.9 (2.45.1) $\mathrm{mg} / \mathrm{dL}$, albumin level was 4.1 (3.5-5.3) g / dL, PTH level was 237 (12-88) pg / mL, alkaline phosphatase was 99 IU/L (60-105 IU/L), $25 \mathrm{OH}$ vitamin D level was $6.4 \mathrm{ng} / \mathrm{mL}$ (20-30 ng/mL) , 24-hour urine calcium was $483 \mathrm{mg} /$ day, creatinine clearance was $75 \mathrm{ml} /$ minute, and prolactin level was $26 \mathrm{ng} / \mathrm{mL}(5-26 \mathrm{ng} / \mathrm{mL})$. Intravenous saline and furosemide treatment was administered. Bone densitometry measurement was showed osteoporosis. Neck ultrasound imaging revealed no abnormality in the thyroid gland with bilateral smooth, ovoid, hypoechoic lesion considered parathyroid adenomas at inferior contiguity of thyroid gland in size of $15 * 11 * 8 \mathrm{~mm}$ on the right and $18 * 12 * 7 \mathrm{~mm}$ on the left side (Figure 1). Tc99m-MIBI scan detected parathyroid adenoma in the lower part of the left thyroid lobe consistent with radioactivity uptake, while in the lower part of the right thyroid was suspicious (Figure 2). PTH level from fine needle aspiration material of parathyroid washout was measured $2097 \mathrm{pg} / \mathrm{mL}$ from the right side and $1989 \mathrm{pg} / \mathrm{ml}$ from left side. Hence, bilateral minimally invasive parathyroidectomy was intended to be performed, but the surgeon saw a third adenoma at retrosternal location by using intraoperative ultrasonography (US), all were removed and pathology report was consistent with parathyroid adenoma (Figure 3 ).

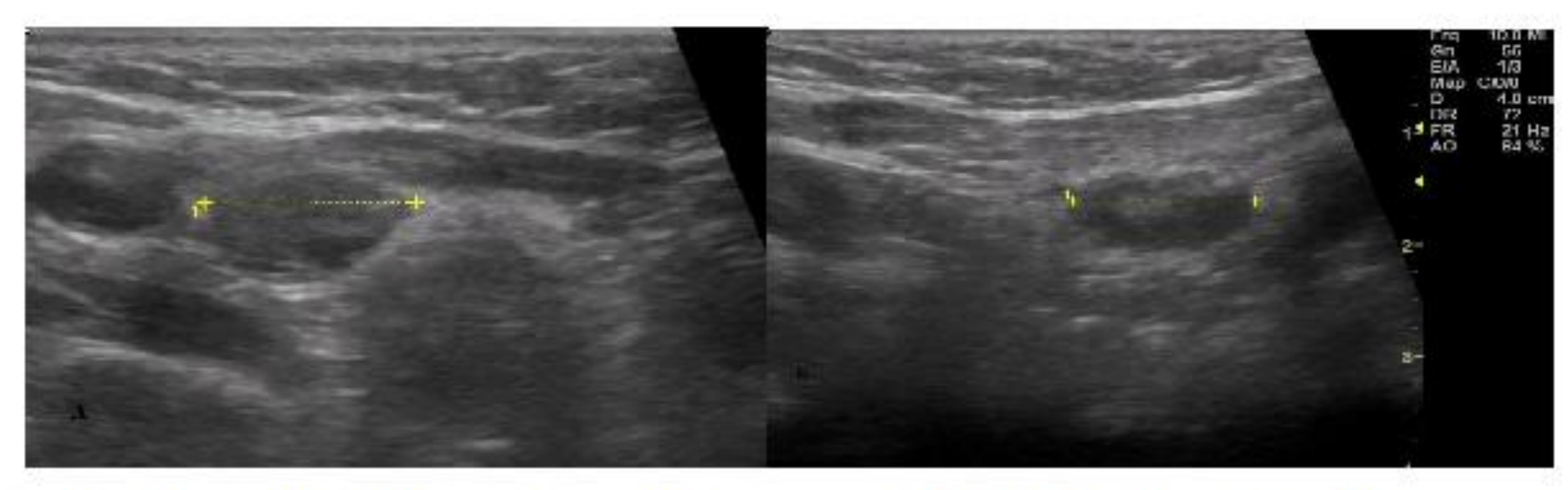

Figure 1: Right parathyroid adenoma in A image and left parathyroid adenoma in B image.

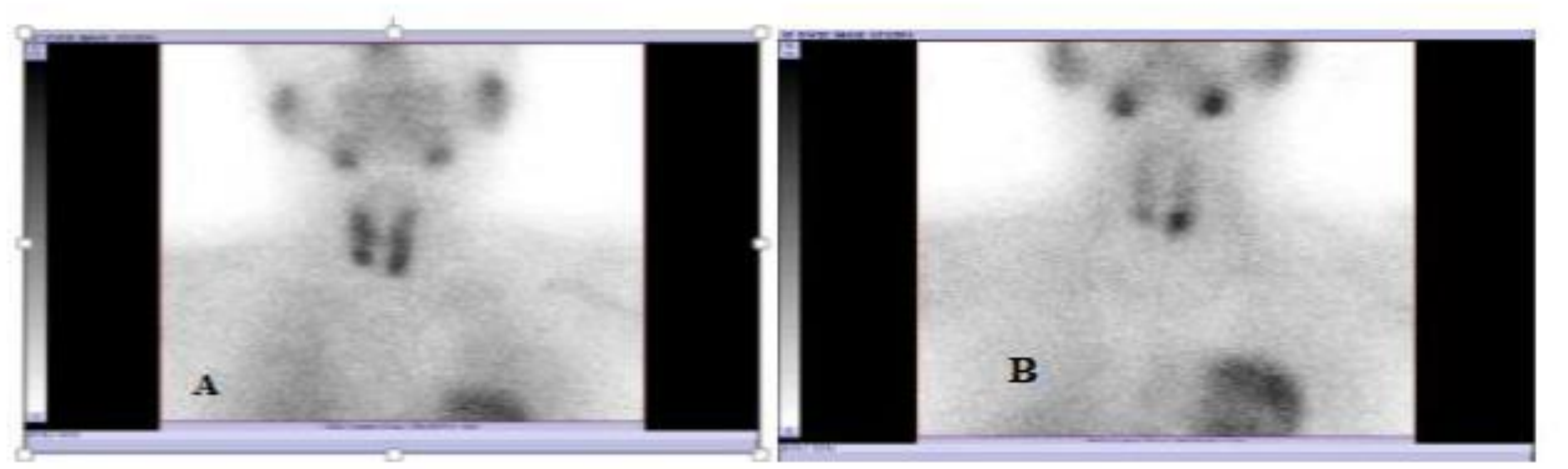

Figure 2: Early phase of parathyroid scintigraphy in A image and late phase of parathyroid scintigraphy in B image

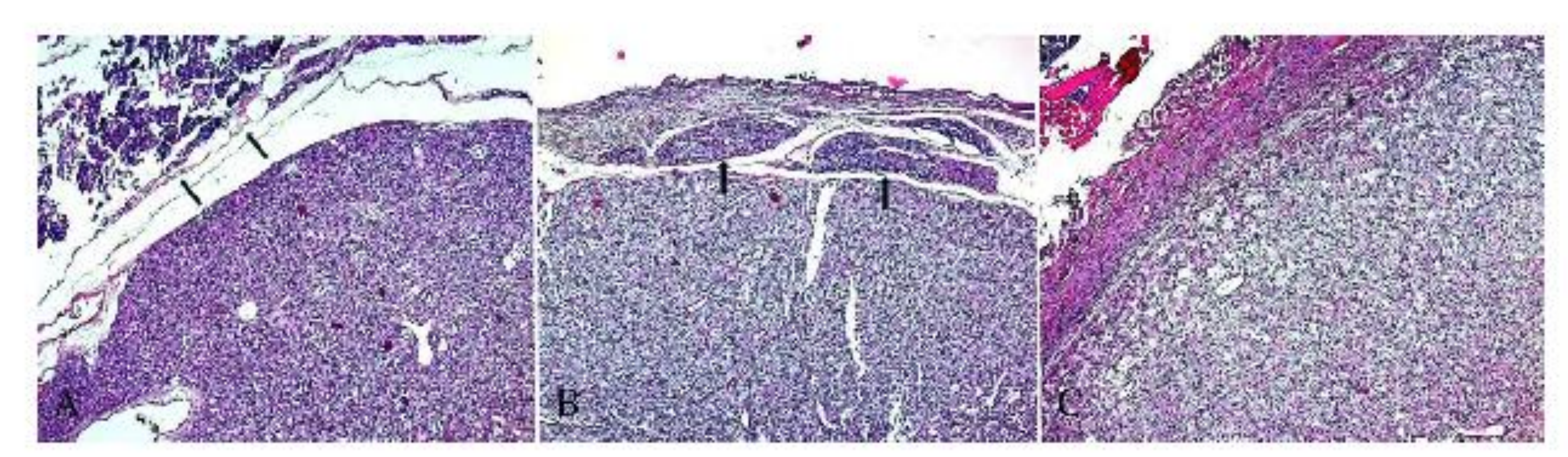

Figure 3: Three parathyroid adenomas predominantly composed of chief cells $(A)$ and clear cells (B and C). Note the normal parathyroid tissue on the top left in A (thin arrows) and compressed normal parathyroid remnants on the top in B (thick arrows).

\section{Conclusion}

Intraoperative parathyroid evaluation by US plays an important role preventing patient from unnecessary surgery 